\section{Cahiers de Narratologie}

Analyse et théorie narratives

$10.2 \mid 2001$

La voix narrative

\title{
À narrateur omniscient lecteur sans voix
}

\section{Aline Mura-Brunel}

\section{OpenEdition}

Journals

Édition électronique

URL : http://journals.openedition.org/narratologie/10242

DOI : 10.4000/narratologie. 10242

ISSN : 1765-307X

Éditeur

LIRCES

Édition imprimée

Date de publication : 1 janvier 2001

Pagination : 295-307

ISBN : 2914561032

ISSN : 0993-8516

\section{Référence électronique}

Aline Mura-Brunel, «À narrateur omniscient lecteur sans voix », Cahiers de Narratologie [En ligne], 10.2 | 2001, mis en ligne le 01 janvier 2001, consulté le 11 juin 2020. URL : http://journals.openedition.org/ narratologie/10242 ; DOI : https://doi.org/10.4000/narratologie.10242 


\title{
À NARRATEUR OMNISCIENT LECTEUR SANS VOIX...
}

\author{
Aline MURA-BRUNEL \\ Université de Pau
}

L'illustre préambule balzacien de La Recherche de l'Absolu laisse le lecteur sans voix : " peut-être faut-il établir dans l'intérêt des écrivains la nécessité de ces préparations didactiques contre lesquelles protestent certaines personnes ignorantes et voraces qui voudraient des émotions sans en subir les principes générateurs, la fleur sans la graine, l'enfant sans la gestation ». ${ }^{1}$ Pris en flagrant délit de précipitation voire d'incompréhension, - et ce, tout au long de La Comédie humaine -, le lecteur s'efforce, autant que faire se peut, de déjouer les prévisions du narrateur. Celui-ci, en effet, doté d'omniscience, tente de conserver la mainmise sur les réactions éventuelles du destinataire et stipule, en contrepoint, les données d'une réception idéale. Son pouvoir, toutefois, génère ses propres limites dans la mesure où il s'appuie sur l'écoute, même imparfaite, de tels propos. Les déficiences de l'accueil entrent ainsi dans le procès scripturaire. Il arrive, de surcroît, que le lecteur ne souscrive pas aux recommandations et admonestations proférées à son intention et cherche même à circonscrire un espace qui lui soit propre. Ainsi l'imagine-ton se projeter dans un lieu laissé vacant par le récit, se frayer une voie dans les brèches éventuelles de la diégèse. Il devient notamment l'autre, celui qui échappe provisoirement aux stratégies auctoriales : il est, par exemple dans La Muse du département, le troisième lecteur d'Adolphe, celui que le texte n'a pas prévu.

La question qui se pose, en termes narratologiques, est double : la maîtrise avérée du narrateur omniscient n'est-elle pas faillible? Ne suscite-t-elle pas les résistances consignées

1 Honoré de BALZAC, La Comédie humaine, La Recherche de l'Absolu, Bibliothèque de la Pléiade, Gallimard, 1979, tome X, p. 657. 
ici et ne produit-elle pas finalement ce lecteur-transfuge qui garde ses distances et garantit l'intégrité fictionnelle de l'écriture romanesque? D'autre part, des récits du XXe siècle comme ceux de Louis-René des Forêts ou de Pascal Quignard qui entretiennent avec le lecteur un rapport paradoxal ${ }^{2}$, ne provoquent-ils pas le même comportement lectoral, alors que la figure du narrateur omniscient a depuis longtemps disparu ? Autrement dit, la suprématie du narrateur n'est peut-être pas le corrélât obligé d'une lecture à contre-courant. L'élaboration d'un espace " libre » où les silences ont un sens ne constituent-ils pas les principes fondateurs de l'acte de lire ? « Lire, c'est créer peut-être à deux ", déclare Balzac dans La Physiologie du mariage, à condition que la retenue puisse côtoyer l'excès.

\section{Le concert de voix}

Des voix entrecroisées ou contradictoires, émanant du narrateur ou de ses substituts, se partagent le pouvoir dans le dispositif narratif. Le narrateur omniscient cède la parole à des personnages délégués, plus ou moins distants, du discours auctorial, tel qu'il se produit dans les préfaces, les articles de journaux et nombre d'incipit romanesques. Au jeu polyphonique qui vient fréquemment obérer le discours monodique s'ajoutent des figures au statut incertain, organisant le récit en maîtres d'œuvre accomplis et éclipsant alors le narrateur principal. Ainsi en est-il de Camille Maupin, l'un des seuls grands écrivains de La Comédie humaine qui, dans Béatrix, gravit les échelons d'une hiérarchie imaginaire : promue du rang de "personnage secondaire » à celui de personnage majeur dans la joute qui l'oppose à la marquise de Rochefide, elle occupe enfin les lieux et places du narrateur en façonnant le destin de Calyste du Guénic et en se livrant à des réflexions de portée générale. Après avoir tout arrangé et tout prévu, Mlle des Touches se retire dans un couvent: "Ma voix arrivera, si mes calculs ne m'ont pas trompée, au milieu d'une cérémonie à laquelle il m'était impossible d'assister ». Tel

${ }^{2}$ Nous pensons en particulier au Bavard de Louis-René des Forêts ainsi qu'au Lecteur de Pascal Quignard. 
l'artiste, elle continue de vivre par l'entremise de ses «personnages »:

Permettez, poursuit-elle, au pauvre Camille qui n'est plus, d'être pour un peu dans le bonheur matériel dont vous jouirez tous les jours. Laissez-moi, cher, être quelque chose comme un parfum dans les fleurs de votre vie, m'y mêler à jamais sans être importune. ${ }^{3}$

Même absente, sa voix couvre les autres voix.

Ailleurs, le lecteur - apostrophé et morigéné - revendique un droit à la parole. Interpellé dans Clotilde de Lusignan (1822), comme il le fut par Diderot dans Jacques le fataliste, il risque de se rebiffer: "Soyez-en juges, chers et précieux lecteurs surtout, ne vous endormez pas! ou dormez si vous gardez le titre de juges. ${ }^{4}$ Humilié à la fin de Louis Lambert (1832), il s'efforce probablement de démentir les propos tour à tour véhéments et ironiques du narrateur : présentant les pensées du jeune philosophe, le porte-parole de l'auteur constate que "entre ces deux fragments, il est une corrélation évidente aux yeux des personnes, assez rares d'ailleurs, qui se plaisent à plonger dans ces sortes de gouffres intellectuels $» .^{5}$ Reste la possibilité de se laisser emporter par "l'extase contagieuse " qui envahit le condisciple de Louis. "Je ne puis expliquer l'effet produit sur moi par cette parole, par le son de cette voix tant aimée ».6 Présence du personnage, ethos restitué par les inflexions de la voix, ravissement du destinataire qui est aussi le narrateur, attention redoublée du lecteur : or, cette chaîne de réactions est encore soumise à la loi du narrateur omniscient, qui provoque le lecteur et lui dicte à son insu ce sursaut de dignité. Il ne peut se passer d'une collaboration jugée pourtant déficiente. Or, même si le narrateur s'efface au profit

${ }^{3}$ H. de BALZAC, Etudes de mours, Béatrix, tome II, Bibliothèque de la Pléiade, Gallimard, 1976, p. 840. Nous avons analysé ce personnage dans notre essai, Béatrix ou la logique des contraires, Champion, 1997, pp. 214-285.

${ }^{4}$ Premiers romans, Clotilde de Lusignan, Laffont, 1999, p. 534.

${ }^{5}$ Etudes philosophiques, Louis Lambert, Bibliothèque de la Pléiade, tome XI, Gallimard, 1980, p. 689. Nous soulignons.

6 op. cit., p. 682. 
d'un personnage qui engage directement le dialogue avec le lecteur, si celui-ci est congédié explicitement («Allons, Messieurs, puisque je vous dis que je ne retiens personne $! \gg^{7}$ ), il louvoie entre le désir de suivre ces injonctions et celui de les dépasser. Comment échapper à la logique du récit, sans le trahir ? Comment faire entendre sa voix, sans être encore celui que le texte a prévu ? Comment écouter ce concert de voix, ce chant des sirènes sans le suivre aveuglément tout en le prolongeant et le réinventant?

\section{Une autre voix[e]}

Il ne s'agit pas de se perdre dans les méandres des intentions d'auteur mais de voir quels types de lectures permettent l'autorité énonciative, quels écarts elle produit ? En imposant une voix et en dessinant les contours d'un lecteur-modèle, le narrateur balzacien provoque des réactions de rejet ou de soumission. En proposant un éventail d'interprétations, il ouvre le récit et favorise un discours interstitiel ou déclenche d'autres lectures. Dans la défaillance de la voix narrative et dans ses excès, s'insère une lecture à contrecourant éminemment créatrice.

La genèse de La Muse du département est, on le sait, tourmentée et fragmentée. Balzac écrit la seconde partie du roman, après avoir relu Adolphe, en 1843. Il confie à Madame Hanska : "J'espère que dans la fin de La Muse, on verra le sujet d'Adolphe, traitée du côté réel». Et le romancier d'inclure dans son texte, des lectures divergentes du roman de Benjamin Constant. Lorsque Dinah de la Baudraye lit Adolphe à travers le prisme de sa propre vie, elle est en quelque sorte piégée (lue dirait Michel Picard). Se voulant lectant, elle n'est somme toute qu'un liseur voire un $l u^{8}$. Au lieu de s'en tenir à une distance critique, elle est impliquée et dévoilée : «Le roman d'Adolphe était sa Bible, elle l'étudiait ; car, par-dessus toutes choses, elle ne voulait pas être

7 Louis-René des FORÊTS, Le Bavard, Gallimard, 1947; 1973, p. 160. Nous reviendrons sur ce point.

${ }^{8}$ Se référer pour ces distinctions à Michel PICARD, La Lecture comme jeu, Minuit, 1986, pp. 112-114. 
Ellénore $» .^{9}$ Or, Lousteau intervient dans cette logique herméneutique et propose sa version des faits présentée par la voix narrative comme définitive :

Vous avez beaucoup lu le livre de Benjamin Constant, et vous avez même étudié le dernier article qu'on a fait. Mais vous l'avez lu avec des yeux de femme. Quoique vous ayez une de ces belles intelligences qui feraient la fortune d'un poète, vous n'avez pas osé vous mettre au point de vue des hommes. Ce livre, ma chère, a les deux sexes. ${ }^{10}$

L'exégèse, développée dans le texte, est admise comme interprétation objective et unique. Et, loin d'entraver le flux narratif, elle s'y mêle si bien qu'elle introduit une scène de rupture en règle, par références interposées. Et Lousteau, porte-parole de la voix narrative, puisqu'il va clore le débat, poursuit l'analyse :

Ce qui tue ce pauvre garçon, ma chère, c'est d'avoir perdu son avenir pour une femme ; de n'avoir rien été de ce qu'il pouvait devenir, ni ambassadeur, ni ministre, ni chambellan, ni poète, ni riche. Il a donné six ans de son énergie, au moment de la vie où l'homme peut accepter les rudesses d'un apprentissage quelconque, à une jupe qu'il devance dans la carrière de l'ingratitude. ${ }^{.1}$

Interprétation partiale que le narrateur impose : c'est la version masculine, malgré la médiocrité du personnage attestée ailleurs, qui tient lieu de vérité. La synecdoque est une condamnation sans appel : il serait sans doute aisé de contrer cette position, mais là n'est pas le propos. Balzac toutefois lève les digues du sens et fraie une voie pour d'autres lectures. Le discours heuristique devient alors un argument déterminant dans le drame affectif qui oppose Lousteau et Dinah. Bel exemple d'intertexte devenu texte, d'ouverture de l'espace diégétique et, pour tout dire, de récupération magistrale des voix dispersées. Autrement dit, le

${ }^{9}$ H. de BALZAC, Etudes de mours, La Muse du département, tome IV, Bibliothèque de la Pléiade, Gallimard, 1976, p. 780.

10 Ibidem.
${ }^{11}$ Ibidem. 
modèle retourné et détourné vient à son tour détourner le sens à son profit. La narration n'en finit pas d'inventer des sursauts.

\section{Le livre du lecteur}

Face à cette double lecture d'un texte invité, le lecteur de chair se retrouve sans voix - perplexe et même défait ; son rôle lui a été dérobé. Outre la voix narrative qui prévaut et impose le sens dans le roman balzacien, interviennent ici deux personnages - lecteurs attentifs et commentateurs diserts. Quelle voix faire entendre ? Quelle voie emprunter? Le chemin tracé par ces voix entrecroisées serait celui d'une coïncidence entre le point de vue de Lousteau - conforté par la voix du narrateur et celui de Dinah en guise de contrepoint. Mais le lecteur balzacien est coutumier des écarts et des transgressions. Sans aller jusqu'à lire «Balzac contre Balzac », il serait peut-être opportun de relancer la dynamique narrative en jouant la carte des intertextes, en dispersant plus encore les signes et leur sens. Le lecteur sort de son aphasie en percevant sous les controverses un espace vacant sinon vierge. L'œuvre manquante qui se dessine en filigrane derrière ces propos ne serait-elle pas celle qui évite ces pièges et ces insuffisances et qui, peut-être se confond avec la production littéraire fictive de Camille Maupin ? En effet, Camille comme Dinah se réfère constamment à Ellénore et s'en détache afin de recouvrer une forme d'innocence, voulant échapper au sort commun, aux lois qui régissent la vie des femmes. Corinne, l'héroïne éponyme du roman de $\mathrm{Mme}$ de Staël, est prisonnière du même carcan et elle est punie pour s'être libérée des préjugés de la société. Or, les modèles, bien qu'obsolètes, rattrapent les personnages balzaciens, textuellement parlant. Qu'il s'agisse d'une mention ponctuelle, comme c'est le cas dans Béatrix, ou d'une comparaison suivie à l'instar de celle que fait Madame de La Baudraye, la destinée éludée réapparaît. Echo subtil entre les romans, Adolphe est évoqué, dans un autre contexte, dans le duel qui oppose Camille à Béatrix : «Je te l'avoue avec la lâcheté de la passion vraie : me l'arracher, ce serait me tuer. ADOLPHE, cet épouvantable livre de Benjamin Constant ne nous a dit que 
les douleurs d'Adolphe, mais celles de la femme ? »12 Camille les a ressenties, le romancier les a transcrites, Camille les a peut-être exprimées mieux que quiconque dans son œuvre. De fait, le romancier prend lui aussi ses distances avec son prédécesseur puisqu'il exhibe les souffrances tues. Camille ajoute en effet, à propos de l'auteur d'Adolphe: «il ne les a pas assez observées pour nous les peindre ; et quelle femme oserait les révéler, elles déshonoreraient notre sexe, elles en humilieraient les vertus, elles en étendraient les vices $»{ }^{13}$ Balzac cependant laisse entrevoir qu'il (ou elle) fait des tentatives et des incursions dans les domaines interdits. $\mathrm{Ce}$ livre, imaginé, reconstruit par un lecteur attentif mais peut-être frondeur, se confondrait alors avec «l'œuvre de Camille », évoquée à plusieurs reprises quoique maintenue dans le silence de l'extradiégèse. Textes dramatiques certes, mais qui font aussi partie des projets de Balzac réinventés dans le roman. Textes rêvés plus qu'écrits par Balzac-androgyne, explorateur de l'être.

Or ce livre reste silencieux, c'est le livre vers lequel tend l'œuvre produite au grand jour, qui se superpose sur le livre écrit, qui le double et l'accompagne, qui en trace les contours et peut-être lui dicte sa loi. Sans faire de Balzac un Mallarmé, il y a là, semble-t-il, un aveu, celui qui consiste à laisser transparaître le cœur du cœur, le livre nécessairement absent mais quintessenciel. Rappelons les propos du narrateur dans Béatrix : "Ce livre, d'un dangereux exemple, fut mis à côté d'Adolphe, horrible lamentation dont la contrepartie se trouvait dans l'œuvre de Camille ". ${ }^{14}$ S'agit-il d'une mise en scène de personnages situés aux antipodes d'Ellénore et qui pourtant disent la solitude de la femme, sa peur de l'abandon et du vieillissement, sa conscience de la mort? «Deux volumes de pièces de théâtre ", " un petit roman admirable, un des chefs-d'œuvre de l'époque » ont fait la gloire de Melle Maupin et l'ont mise au ban de l'amour. Ce livre fictif (même si l'on trouve des correspondants effectifs, qu'il s'agisse des romans de George Sand ou de Théâtre de Clara Gazul de

${ }^{12}$ Etudes de mours, Béatrix, tome II, p. 773.

13 Ibidem.

14 Op. cit., p. 699. 
Prosper Mérimée) bénéficie des erreurs d'Ellénore et peut-être de l'expérience de Camille - sans parler de l'épisode biographique avec Laure de Berny : il ne dévoile que ce qu'il n'est pas. Négatif troublant qui hante le récit balzacien, à l'instar du "livre jaune" - A Rebours - dans Le Portrait de Dorian Gray, il ne cesse d'interroger le lecteur. Et, c'est ce trouble, cette incertitude, cette méconnaissance même qui lui confère une existence fictionnelle et fonctionnelle. Il lui rend sa voix et lui offre un espace de parole. Le romancier ne fait-il pas de ce texte une sorte d'exemplum ? Ecrire qu'il est écrit, n'est-ce pas dans une certaine mesure l'avoir écrit mais aussi le donner à lire et à écrire ?

Ainsi la voix narrative s'est-elle démultipliée, annihilant sans espoir de retour le régime monodique ; elle a implosé sous la pression de plusieurs personnages-relais puis elle a ouvert une brèche, celle dans laquelle s'engouffre le lecteur autrefois muet. Le narrateur n'assigne plus de limites aux interprétations ; il fait éclore ces discours de l'autre sur l'autre - le livre absent et pourtant nommé, qui réorganise le système axiologique et narratologique dans Béatrix et dans La Muse. Il est vrai que ce dernier texte, à la genèse complexe, autorise plus qu'un autre sans doute ces inserts et ces transgressions. Mais il semble que l'édifice balzacien ne laisse de montrer ses fragilités et ses incertitudes : la figure du narrateur omniscient vacille, les personnages prennent leur indépendance et le lecteur averti ou perverti recouvre la voix que le texte étouffait tout en affirmant son absolue nécessité. Le lecteur se glisse alors dans les silences du texte.

\section{Les silences du texte}

Dans La Muse du département, le lecteur a le loisir de s'immiscer dans les failles du discours. Sans parler des dysfonctionnements dus aux ajouts successifs, il existe des silences dont les personnages font état pour se démarquer du roman lu et relu, Adolphe. Lousteau, jouant de la prétérition, déclare à sa maîtresse : " Il est des Adolphes qui font grâce à leur Ellénore des querelles déshonorantes, des plaintes, et qui se disent : Je ne parlerai pas de ce que $\mathrm{j}$ 'ai perdu $» .{ }^{15}$ Il utilise

${ }^{15}$ La Muse du département, p. 780. 
ainsi son silence comme un argument en faveur de sa cause et relit son passé avec complaisance ; le lecteur de chair évalue à son tour ce silence et mesure l'écart qui sépare les deux personnages, le discours du phraseur et les faits consignés précédemment dans la narration. Quant à Dinah, avec plus de justesse sans doute, elle déclare : "Mon silence ne sera pas une plainte et ma parole ne sera pas une querelle ". ${ }^{16} \mathrm{Un}$ silence muet s'opposerait à un silence paradoxalement éloquent. Dans ce jeu du faux palimpseste, le lecteur comble les silences du texte et fait entendre une autre voix, constituée des tensions entre les divers comportements. Il s'agit là encore d'une forme de silence qui reste soumis à un artifice rhétorique, dans la mesure où il est rendu explicite et s'inscrit dans la logique d'un récit qui est a priori du discours plutôt que du silence.

Outre ce procédé de mise en relief par rapport à une parole usée ou délétère, le silence constitue aussi un langage et un choix existentiel. Ainsi Camille a-t-elle choisi le silence lorsqu'elle n'avoue pas d'emblée sa stratégie à Béatrix et, plus encore, lorsqu'elle se tait après avoir tant discouru et tant écrit, entre les murs d'un cloître. C'est là que le lecteur joue un rôle actif, animant les moments de latence, donnant la parole aux absents et réparant les lacunes: il peut alors retrouver la voix ensevelie de Camille, imaginer l'œuvre écrite (qui reste non-dite) et lui conférer une légitimité. Fragment mort du texte qui se construit à la lumière des propos tenus, des projets de Balzac et des rêves du lecteur: de la pièce manquante procède en partie le livre dont l'absence structure l'œuvre, à l'instar des " débris de pensées », à la fin de Louis Lambert, issus d'un Traité de la volonté écrit par Balzac.

Le poète aussi s'est effacé dans La Comédie humaine pour laisser éclore le romancier, historien et visionnaire. Balzac réécrit sur ses papiers noircis et ensevelis sous les intrigues romanesques. Lucien Chardon en est la preuve irréfutable. Les autres intertextes - comme le roman de B. Constant - ne fonctionnement pas différemment, du point de vue de la logique narrative. Ils procèdent toujours d'un double mouvement de rejet et d'insertion : relégués dans le

${ }^{16}$ Op. cit., p. 775 . 
silence, ils réapparaissent transformés et destinés à d'autres usages. Ainsi en est-il du recueil des Marguerites qui devient l'emblème d'un renoncement consenti et alimente le comportement du jeune homme. Il n'écrit plus de poèmes mais vit le roman d'un poète et, même lorsqu'il se renie, il reste l'être inadapté et fragile exilé de la cité. Et le lecteur retrouve pas à pas la trace de ce chant palinodique qui informe le tramé narratif.

Parfois au contraire, le texte - surdéterminé - ne ménage plus aucune plage de silence : il déborde de détails et de sens ; il comble les vides et les espaces morts, réduisant à néant les charmes de l'implicite. Il ne reste alors plus d'autres positions de lectures possibles que l'adhésion complète et muette, ou le repli et même la fuite, ou encore la violation des sens obvies. Tour à tour, le lecteur emprunte ces chemins divergents. Subjugué par les aveux prolixes et lyriques de Félix dans le Lys, lassé peut-être par les admonestations réitérées, il laisse «tomber le livre des mains », comme le redoute l'ami de Louis Lambert, avant de le reprendre avec un nouveau souffle. Il choisit alors d'outrepasser ses droits, il impose une voix lectorale et lit - dans les failles du discours - des livres dans le livre. Cela aussi sans doute, le narrateur balzacien l'avait imaginé ou même attendu, mais l'histoire ne le dit pas.

Il arrive également que les romans du XXe siècle offrent des exemples de discours surabondants qui laissent le lecteur sans voix. Le lecteur du Bavard de Louis-René des Forêts, de La Chute d'Albert Camus (sans parler, chez Balzac encore, de L'Illustre Gaudissart), submergé par l'afflux de paroles, recherche l'ombre discrète, le coin solitaire où se recueillir ; il se révolte contre les discours prolixes qui lui dictent le laconisme complaisant ou l'enthousiasme discret ; il invente, dans le texte, une " aire » inviolée qui lui confère identité et légitimité. Mais cette reconquête ne va pas de soi. Dans $L e$ Bavard (1947), le $i l$ ne recouvre pas une entité homogène : à la polyphonie de la voix narrative correspond une polyphonie des instances lectorales. Le je se mue en un $i l$, et cette personne clivée s'adresse à un destinataire non unifié, scindé en plusieurs figures possibles, sous l'effet de discours décousus. Ainsi l'auditrice fortuite du dancing - narrataire 
dépourvue de voix mais dotée d'un rire sarcastique - cède-telle la place à un vous versatile : "il me paraît nécessaire de vous donner une idée sommaire du décor». Resurgit alors une distinction bien connue entre un narrataire intradiégétique et un autre extradiégétique. Il serait en l'occurrence peut-être plus judicieux de parler de lecteur impliqué ou inscrit, selon la terminologie de Jean Rousset ${ }^{17}$. Ce personnage fictif de plus établit un relais avec le récepteur effectif que nous sommes. Tous sont contraints à une forme de mutisme, même si le silence ne dure que le temps que s'étanche « la soif oratoire » 18 du bavard. Le lecteur interpellé par la voix narrative - qui assure parfaitement à ce moment-là sa fonction de régie - subit le flot de paroles jusqu'à ce qu'il soit congédié : la clausule est sans détours. A cette " frontière du récit », le lecteur de chair se sent lui aussi apostrophé par l'injonction du narrateur ${ }^{19}$. Avant de disparaître, le récepteur interne est successivement désigné par l'embrayeur "on » puis « vous », accentué par le nom « Messieurs ».

Dans l'ensemble je suis arrivé à ce que je voulais obtenir. Je me suis soulagé, et qu'on ne me dise pas que ce n'était pas la peine. Or, maintenant, je suis las. Allons, Messieurs, puisque je vous dis que je ne retiens plus personne !20

Une certaine porosité des limites textuelles produit la multiplication des voix lectorales convoquées et leur confusion. S'instaure aussi une incertitude quant à la source de la parole : s'agit-il toujours du bavard ou d'une instance narrative plus large, proche de l'auteur? Cela reste indécidable ; et la fluctuation entre à la fois dans la composition et la réception de ce récit, précurseur de l'écriture dite impassible. Toujours est-il que le lecteur réel a du mal à s'immiscer dans les interstices du texte : il s'efforce de s'écarter de la position assignée au lecteur inscrit, mais il ne saurait aisément créer son

17 Jean ROUSSET, Le Lecteur intime, Corti, 1986.

18 Louis-René des FORÊTS, Le Bavard, p. 16.

19 Se référer sur ce point à notre article «Les derniers mots au lecteur ", in Les Frontières du récit, Narratologie n 3, 1998.

${ }^{20}$ Louis-René des FORÊTS, op. cit., p. 160. 
espace de parole, après avoir vu le verbe ainsi dévalorisé et ravalé au rang de maladie et de crise. Seule l'écoute muette semble souhaitable. La lecture active n'est jamais qu'une interruption et la voix du lecteur un avatar de discours superfétatoires, une pathologie de la réception. Pour sortir de l'aporie et ne pas suivre le modèle de lecture induit par le texte, il convient peut-être de procéder là aussi à une forme de lecture à contre-courant.

Il n'est plus question pourtant aujourd'hui de narrateurs omniscients, mais de personnages souvent dérisoires qui n'ont pas su garder le silence et disent la vanité de la parole qu'ils galvaudent et trahissent, au moment où elle s'énonce. Or, l'autorité de la voix énonciative reste intacte. Elle sème le trouble quant à la désignation des destinataires apostrophés : les changements inopinés de pronoms font vaciller le statut d'instances fluctuantes; en revanche, les figures du lecteurs convoqués par le roman balzacien conservent une apparence d'homogénéité. Sans doute faut-il s'éloigner du texte du Bavard pour le retrouver et le décrire? La distance permet de rejoindre le sens d'un récit - qui n'est que l'écho d'une voix n'ayant d'autre identité que celle d'un silence rompu, à l'instar de l'écriture elle-même. Le lecteur réel doit donc se distinguer de ce lecteur impliqué que la voix narrative interpelle et assujettit provisoirement à sa loi. Echappant à cet espace surchargé de mots jusqu'à l'asphyxie, le lecteur dévoile l'imposture - présentée comme telle dans le récit - de ces discours dilatés. Il peut dire et écrire. On le sait, ces textes ont donné lieu à d'admirables commentaires, qu'il s'agisse de «La Parole vaine » de Maurice Blanchot, du Vou de silence de Pascal Quignard ou des essais de Dominique Rabaté21.

Et pourtant, la tentation est grande, celle qui consiste à se taire, recueilli, face à l'œuvre qui nous submerge :

21 Maurice BLANCHOT, "La Parole vaine", postface, édition Gallimard, 1963 ; Pascal QUIGNARD, Le Vøu de silence, Fata MORGANA, 1985 ; Dominique RABATÉ, Louis-René des Forêts. La Voix et le volume, Corti, 1991 et Poétiques de la voix, Corti, 1999. 
Ah lecteur. Comprendrez-vous que vous décidant pour de bon à arracher ces pages jusqu'à la dernière de ces pages, et coupant court à cette rhétorique de miroirs où nul reflet franchement ne s'inscrit ni de moi ni de vous $[\ldots]$, nous nous tairions. ${ }^{22}$

Or, le silence, en l'occurrence, est impossible. En prônant la valeur du silence, nous émettons encore des sons, nous produisons des textes. Nous parlons, nous écrivons, nous lisons à haute voix, soumis à une nécessité peut-être fatale ou simplement importune. Le livre écrit nous incite à le dire, le transformer, le réécrire. Nous nous engouffrons dans ses silences, nous écoutons sa voix et nos voix en écho. Absent ou imposant, le narrateur produit ces effets, ces discours. Chance ou imposture, la voix du lecteur s'est, ici, une fois de plus fait entendre...

Somme toute, la figure omnisciente ou intermittente qui se déploie dans l'édifice balzacien ne compromet pas l'émergence d'une autre voix qui retentit face à celle tonitruante des ténors de la narration. Quant au bavardage de personnages en déroute que ne guide nul narrateur organisateur, il plonge le lecteur dans le désarroi avant que celui-ci ne comprenne que les abîmes ouverts par ces flots de paroles déversées ou contenues supposent et appellent des fragments de réponses ou de nouvelles divagations. Le narrateur, qu'il soit discret ou disert, déclenche l'intervention du lecteur. Nous ne dirons rien des récits de Marguerite Duras - pour ne pas allonger le propos -, mais nul n'ignore que le choix d'une écriture elliptique, lacunaire et pour tout dire éloquemment silencieuse, suscite un discours qui s'avère supplétif et marginal avant de progresser vers la construction d'un sens. Le grand œuvre se reconnaît peut-être aussi à sa faculté d'admettre l'intrus, lui laissant prendre la parole et le pouvoir, sans se déliter ni disparaître. Quelles que soient la forme de la narration et la force de la voix narrative, le lecteur s'immisce dans les zones d'ombre et de silence (qu'il crée lui-même parfois), croyant assurément que sa parole, dotée d'une indéniable puissance palingénésique, relance la dynamique créatrice et revêt une grâce épiphanique.

22 Pascal QUIGNARD, Le Lecteur, Gallimard, 1976, p. 132. 